\title{
La reflexión histórica de la educación: de la transmisión a la tradición*
}

\author{
Historical reflection of education: \\ from transmission to tradition
}

La realidad histórica, dinámica y concretamente considerada, tiene un carácter de praxis que, junto a otros criterios, lleva a la verdad de la realidad y también a la verdad de la interpretación de la realidad.

Ignacio Ellacuría

$\dot{L}$ a realidad es la realidad?

Pilar Garzón Galindo**

Edna Isorin Jiménez Montealegre***

Resumen. Este artículo estudia la educación para la ciudadanía a comienzos del siglo XX en el contexto de una región del norte de Colombia, denominada Caribe y se ubica en uno de sus ocho departamentos, el Departamento de Bolívar cuya capital es Cartagena de Indias. A partir de ésta experiencia se invita a los maestros a pensar

Artículo de reflexión que se enfoca en el estudio de la educación de la ciudadanía a comienzos del siglo XX en la región Caribe de Colombia.

** Licenciada en Filosofía e historia, especialista en Educación en Derechos Humanos, Magister en Ciencias de la Educación y candidata a Magister en Historia. Coordinadora académica Fe y Alegría de la Américas (Cartagena, Colombia). Docente e investigadora, directora de la línea de investigación: Educación e interculturalidad de la Facultad de Educación, Ciencias Humanas y Sociales - Universidad San Buenaventura (Cartagena, Colombia). E-mail: piga14@gamil.com\pgarzon@usbctg.edu.co

*** Licencias en Filosofía y cultura para la Paz, Magister en ciencias de la Educación. Coordinadora académica Escuelas Profesionales Salesianas (Cartagena, Colombia). E-mail: montealegre77@gmail.com 
desde la historia y para la historia, el reto educativo que enfrentan como constructores de la historia de la educación en el ámbito regional a comienzos del siglo XXI. De esta manera se busca despertar la conciencia histórica de los educadores. Para lograr este propósito se plantea la reflexión histórica de la educación desde los tres momentos estructurales de la tradición que propone Ignacio Ellacuría, para interpretar la historia como realidad. Así se encuentra en primer lugar el momento constituyente; en segundo lugar, el momento continuante y en tercer lugar, el momento progrediente. Estos tres momentos estructurales de la tradición permiten comprender la envergadura ética y política de lo que implica hacer parte de una cultura, de un país, de un territorio, de un hacer y -fundamentalmente-, lo que implica ser maestro reproductor de discursos y formas deshumanizantes institucionalizadas por relaciones artificiales de dominación. Si el maestro asume su práctica educativa y pedagógica como tradición y no como transmisión se hará responsable de la realidad que vive en su cotidianidad. Está investigación tomó la historia social para contextualizar la época a través de la observación, la lectura analítica y crítica de documentos escritos por: gobernadores, alcaldes, ministros y demás funcionarios que se pronunciaron frente a la Instrucción pública en el ámbito nacional y local, durante el primer trienio del siglo XX.

Palabras clave. Conciencia histórica, transmisión, tradición, historia como realidad.

Abstract. This paper studies education for citizenship in the early twentieth century in the context of a region of northern Colombia called Caribbean and is located in one of its eight departments, the Department of Bolívar whose capital is Cartagena de Indias. From this experience encourages teachers to think from the story and history, the educational challenge facing as builders in the history of education at the regional level at the beginning of the XXI century. Thus it seeks to raise historical awareness of educators. To this end there is the historical reflection of education from three structural moments of tradition Ignacio Ellacuría proposed to interpret history as reality. This is the first time constituent, secondly, the time continuant and thirdly, the time progrediente. These three structural moments of tradition enable us to understand the ethics and politics of scale which 
means being part of a culture, a country, a territory, a make-andfundamentally, what it means to are master player speeches and forms dehumanizing institutionalized by artificial relations of domination. If the teacher assumes it's educational and pedagogical practice as a tradition and not be responsible transmission of reality in their daily lives. It took research to contextualize social history through the time of observation, analytical and critical reading of papers written by: governors, mayors, ministers and other officials who spoke against public education in the national and local levels for the first three years of the twentieth century.

Keywords. Historical consciousness, transmission, tradition, history and reality.

\section{Introducción}

¿Para qué sirve la historia? Y particularmente ¿̇para qué sirve una investigación de historia de la educación en el Departamento de Bolívar a comienzos del siglo XX? Esta es una de las preguntas que quisiera responder a los maestros que se han interrogado sobre este tema y que se perfila como un buen pretexto para exponer por qué la Historia es una ciencia del Espíritu, que como diría el historiador francés Marc Bloch es una «ciencia de los hombres en el tiempo» contrario a pensar «la historia como ciencia del pasado» ${ }^{1}$.

Se decidió claramente abordar este cuestionamiento a partir de una visión histórica para dar respuesta a la problemática que enfrenta y de la cual carecen en términos científicos los estudios sobre Ciencias de la Educación a nivel regional.

Seguidamente, relacionamos la perspectiva de la historia propuesta por Bloch (1997), con el concepto filosófico de historia acuñado por Ignacio Ellacuría, filósofo y teólogo de la liberación, a quien le preocupaba, finalizando la década del cincuenta, el desmesurado auge de la tecno-

1 Marc Bloch, fue un historiador francés, fundador de la escuela historiográfica de los Annales junto con Lucien Febvre, fusilado por la Gestapo, en 1944. En su producción historiográfica se intereso por el tema económico y social para la mejor comprensión de la Historia como Ciencia Social. 
logía en detrimento del desarrollo integral del ser humano que según él, olvida los verdaderos problemas de la humanidad ${ }^{2}$.

Es importante anotar que el pensamiento de Ellacuría tiene origen en los supuestos biológicos, antropológicos y morales de la filosofía de Xavier Zubiri (estudiante de Husserl y Heidegger), dentro de la categoría de la realidad histórica, realizando así su propia interpretación desde la cual puede hablarse de responsabilidad y liberación, en cuanto transformación. Ellacuría encuentra una estrecha relación entre verdad y liberación, entendida esta última como la búsqueda de la verdad latinoamericana. Dicha liberación cumple con tres funciones fundamentales: en primer lugar la criticidad, entendida como la crítica al orden establecido que desvirtúa y falsea la realidad; segundo la fundamentalidad, que trata de desenmascarar los fundamentos artificiales de las estructuras de poder. Y por último, la función creadora, que consiste en la actitud reflexiva del sujeto frente a la verdad de la realidad para «iluminarla, interpretarla y transformarla» (Ellacuría, 1985, p. 25), es decir para ejercer su función liberadora.

Desde estas tres funciones el maestro como buscador de la verdad, -no sólo de la disciplina que enseña sino de la verdad que él o ella reproduce en los procesos educativos y pedagógicos, es el que puede develarse develando su práctica para escribir la verdad de su acontecer en el tiempo-, que será relatada en los anales de la Historia de la Educación del Siglo XXI como experiencia de construcción política, social, cultural en el ámbito escolar.

De esta manera, se asume el pensamiento de estos hombres de ciencia y en especial acerca de la comprensión filosófica de la historia, como el puente epistemológico a través del cual se une la Historia de la Educación y las Ciencias de la Educación. Para iniciar esta tarea se toman de Ellacuría dos postulados más. Primero: considerar que la historia es el lugar de la ética; y segundo, que el hombre tiene que hacerse cargo de la realidad y cargar con ella, es decir la realidad es su responsabilidad. Así: «En definitiva, la realidad histórica, dinámica y concretamente considerada, tiene un carácter de praxis que, junto a otros criterios, lle-

2 Ignacio Ellacuría fue un sacerdote jesuita de origen vasco, nacionalizado en el Salvador y asesinado durante el período de la Revolución salvadoreña en el año de 1989 por soldados del Ejército Nacional. Siempre le preocupó el destino histórico de los más pobres y desfavorecidos de la humanidad. 
va a la verdad de la realidad y también a la verdad de la interpretación de la realidad» (Ellacuría, 1985, p. 45). Esta concepción de la historia como praxis, recupera el valor que ella tiene como ciencia del Espíritu, para interpretar desde esta orilla los asuntos propios de la historia de la educación en el departamento de Bolívar; historia, de la cual también todos los maestros y maestras somos responsables.

Atendiendo a la responsabilidad social que posee cada maestro y maestra al atreverse a proponer a través de sí un estilo de enseñanza y a pensar la educación como humanización, se propone recuperar la conciencia histórica de los maestros para considerar que «Las ciencias del Espíritu no deben servir sólo para ratificar desde la tradición histórica lo que ya sabemos sobre nosotros mismos, sino también, directamente, para algo distinto: procede recibir de ellas un acicate que nos conduzca más allá de nosotros mismos» (Gadamer, 2010, p. 46). Esto permite afirmar que el conocimiento y la comprensión del pasado ayudarán a los maestros y maestras que hoy hacen parte del sistema educativo, a construir conscientemente la Historia de la Educación del siglo XXI, en el tiempo y la hora de la era digital, para hacer de la educación un proceso de humanización que priorice a la persona en su contexto. Por esta razón, si la historia es la realidad, la realidad del accionar educativo y pedagógico, dará cuenta de lo que hoy es la educación; y este ser educativo y pedagógico del presente sea precisamente lo que ésta generación de maestros entregará al futuro.

El futuro de la realidad educativa y pedagógica del Caribe colombiano depende de un alto índice de criticidad que le permita comprender al maestro que su práctica es palabra. «No hay palabra verdadera que no sea una unión inquebrantable entre acción y reflexión y por ende, que no sea praxis. De ahí que decir la palabra verdadera sea transformar el mundo» (Freire, 2005, p. 105).

De esta manera nos interrogamos sobre ¿Cuál es la palabra verdadera que puede pronunciar el maestro costeño? La que produce su conciencia histórica, es decir, en la medida que se perciba testigo de su historia. Desde allí su conciencia ya no es responsiva sino reflexivamente más responsable de esa historia.

La realidad de la historia educativa en el contexto caribeño es responsabilidad del maestro que habla y escribe, y cómo habla y escribe, deja 
entrever el desenvolvimiento de su espíritu, a través del cual puede rehacer lo hecho descubriendo lo que hace y rehace. La palabra del maestro libera al estudiante de su ignorancia; ignorancia entendida como la no palabra, como incapacidad para el diálogo que niega la oportunidad para ser palabra verdadera en clave de liberación.

Apoyadas en el concepto de historia de la Escuela de los Annales, en los postulados filosóficos de Ellacuría y por último, en la pedagogía de la liberación de Paulo Freire, desarrollamos tres miradas: la histórica, la filosófica y la pedagógica para reconocer que la «historia de los hombres en el tiempo» es «conciencia historiadora» que transforma, al comprender que la «historia es la realidad» para conocerla, vivirla y transformarla es decir, para crear la nueva realidad que emerge de la palabra liberadora (Ellacuría, 1985).

Ellacuría se ocupa de lo humano en cuanto Espíritu que se desenvuelve al objetivarse en la realidad que el mismo crea, esta objetivación del Espíritu en la historia que se traduce como realidad, es lo que permite pensar que la realidad se transforma por ser creación del Espíritu humano que se objetiviza y subjetiviza en un espacio determinado, en el cual las relaciones políticas, económicas, sociales, y por supuesto -educativas y pedagógicas-, resultan no ser siempre universales sino particulares.

La historia como realidad universal y particular está sujeta a la transformación de sí misma, es decir, al cambio entendido como liberación, el cual consiste en comprender que la mediación de la acción educativa no es la transmisión ${ }^{3}$ sino la tradición, «La razón formal de lo histórico está en la tradición y no en la transmisión» (Ellacuría,1991, p. 392). Entendida aquí la tradición como «lo entregado» en la ritualidad de la cotidianidad de lo personal y lo colectivo, así, tal vez la educación sea eso: «lo que se entrega» en la ritualidad de la cotidianidad escolar. Seguidamente, esto permite preguntar: ¿qué están entregando los maestros de comienzos del siglo XXI a esta generación tecnologizada?

Nuestra respuesta se inclina hacia la reflexión histórica de la educación desde los tres momentos estructurales de la tradición que propone

3 La transmisión entendida desde su carácter biológico como génesis de la historia humana, se asume como transmisión tradente. 
Ellacuría para interpretar la historia como realidad. Así se encuentra en primera instancia el momento constituyente; en segundo lugar, el momento continuante y en tercer lugar, el momento progrediente. Estos tres momentos estructurales de la tradición permiten comprender la envergadura ética y política de lo que implica hacer parte de una cultura, de un país, de un territorio y de un hacer. Fundamentalmente también, lo que implica ser maestro reproductor de discursos y formas deshumanizantes institucionalizadas por relaciones artificiales de dominación. Si el maestro asume su práctica educativa y pedagógica como tradición y no como transmisión se hará responsable de la realidad que vive en su cotidianidad educativa y pedagógica.

En primer lugar, el momento constituyente de la tradición se refiere «a la forma de estar en la realidad» (Ellacuría, 1991, p. 392). Cuando el hombre o la mujer nacen reciben una instalación en la realidad que se asume en el «modo de estar» en ella como individualidad y colectividad. Este «modo de estar» es lo recibido y se constituye en tradición que alimenta la conciencia de las individualidades y las colectividades. Desde esta perspectiva afirma Ellacuría, que la humanidad es el sujeto constituyente de la tradición, no por los contenidos tradicionales sino por los modos de estar en la realidad.

Por lo anterior, sostenemos que si la humanidad es el sujeto constituyente de la tradición, se puede afirmar que el maestro, en nombre de la humanidad, es el sujeto constituyente de la historia de la educación. Aquí cabe preguntarse por el qué de la tradición educativa y pedagógica recibida por los maestros y maestras en esta parte del Caribe colombiano; cómo se refleja esta tradición en la educación, en el educador, en la pedagogía, en el pedagogo, en el sistema y en las instituciones educativas.

Si el maestro recibe tradición se hará consciente de lo que recibe para sí, es decir, no hará de su discurso pedagógico un dispositivo dominante para la regulación de la reproducción cultural y educativa; por el contrario con lo que recibe inicia su andar en el tránsito hacia lo nuevo que irrumpe y recrea la mismidad del sujeto que enseña y aprende. Así se conduce al segundo momento, el continuante.

En segundo lugar, el momento continuante de la tradición se refiere a lo acaecido en la subjetividad que recibe, la transformación de lo recibido. No sólo las personas y las poblaciones continúan con lo recibido, 
con lo que se les ha dado, sino que cada persona y población hace que continúe lo recibido, impulsándolo hacia adelante, produciendo cambio, renovación de la historia, a través de su ser, de su praxis. La tradición no es repetición, es novedad que supera el momento constituyente o como bien nos dice el mismo pensador: «el momento continuante de la tradición es así todo menos repetición; la repetición en la historia resulta así absolutamente imposible y la fidelidad histórica no puede lograrse más que en la forma del proseguimiento» (Ellacuría, 1991, p. 393).

El tercer lugar, el momento progrediente de la tradición, el hombre tiene que ir realizando su vida, es decir, apropiándose de ella para optar por una u otra realidad. Es el momento de la conciencia histórica entendida como «la posición reflexiva en la consideración de todo aquello que es entregado por la tradición (...) este comportamiento reflexivo cara a cara de la tradición se llama interpretación». (Gadamer, 2007, p. 43). En este momento el maestro receptor de la tradición que ha recibido es el interprete de la realidad educativa y pedagógica y lo que entrega es su interpretación de ésta realidad, con los cambios que permiten que la tradición continúe en otros.

Hay aquí un principio de dinamismo histórico bien preciso: el carácter específico-social de la tradición lleva consigo forzosamente la necesidad de no poder estar quieto. Este no-poder-estar-quieto es constitutivo de la tradición estructuralmente entendida, tanto por su momento de entrega como por su momento de recepción y su momento de realización. (Ellacuría, 1991, p. 39).

Este no-poder-estar- quieto permite comprender que la educación y la pedagogía tienen tradición e historia. Por ello, cabría preguntarse ¿Cuál es la tradición ética y política de las escuelas públicas qué forman para la ciudadanía y la humanización? Por su naturaleza, debería ser cambiante y reflexiva, dispuesta siempre a renovar su práctica para optar por una realidad siempre nueva y diferente.

En síntesis, reflexionar la Historia de la Educación desde los tres momentos estructurales de la tradición es la tarea que emprendemos para analizar y comprender lo que ha sido el caso concreto del Departamento de Bolívar durante el período que la historiografía colombiana ha denominado Hegemonía Conservadora, de 1903 a 1930. 


\section{El momento constituyente de la tradición educativa y pedagógica de la educación en el Departamento de Bolívar}

La erección de repúblicas emancipadas del poder colonial y la construcción del concepto de ciudadano y ciudadanía son dos elementos que surgen casi al unísono de las transformaciones políticas que se dan en el período conocido como el de la Independencia. El vehículo del que se vale la nueva república, -otrora virreinato de la Nueva Granada-, para consolidar su propósito de nación es el sistema educativo.

Durante el último tercio del siglo XVIII, tras las reformas borbónicas que incursionaron en el Nuevo Reino de Granada, como resultado de profundos cambios culturales que acontecieron en Europa, se vislumbran nuevas perspectivas para entender la realidad política, social y económica de estos territorios americanos que en un sentido práctico y dignificante, pretendían reestructurar la organización de Estado racionalizando sus beneficios. Para esto, fue indispensable implementar en cuanto a lo educativo de nuevos campos del conocimiento científico que le fueran de utilidad y provecho a la grandeza tanto del reino como del imperio. La educación para ese entonces concentrada en las élites criollas transitaba de modelos escolásticos a modelos modernos, producto de las corrientes de pensamiento filosófico ilustrado. Dicho transito tenía pocos adeptos dentro de la sociedad neogranadina, pero a pesar de ello se logro el acercamiento a nuevas formas de entendimiento y comprensión que fueron las bases en cuanto al discurso y sus conceptos de los cambios políticos acontecidos a partir de 1810 (Silva, 2005).

En este contexto se difunden variedad de conceptos que nutren el discurso renovador de la realidad del virreinato y que a la larga se convierten en las razones que motivarían la exigencia de cambios radicales que dieron como resultado procesos de ruptura con los vínculos que se tenían de antaño con la metrópoli. Las élites criollas esforzadas por estimular el patriotismo y la conciencia nacional entre amplios sectores de la población, y para impulsar su identificación con la sociedad y con el nuevo Estado, emplearon el término de ciudadano en el sentido de «miembro del cuerpo estatal», partiendo de la base política surgida de la Declaración de los Derechos del Hombre y del Ciudadano hija de la Revolución francesa, así como de la Independencia norteamericana y las constituciones que se levantaron (Köning, 1994). Cabe anotar que durante el periodo 
colonial, el término ciudadano había estado relacionado con términos bastante corrientes como el de vecino y vasallo, en relación a su condición de habitante de una ciudad, amo de casa y elegible para el cabildo, como a la condición de súbdito ante el Rey español, respectivamente. El término fue adaptado y empleado a las nuevas circunstancias, con plena conciencia en cuanto al título y el status del nuevo ser ciudadano. En todo caso, el uso del término ciudadano en referencia a su nuevo significado como status político, sólo ocurrió después de 1808, cuando los acontecimientos tanto internacionales como nacionales bullían en procesos de revoluciones.

La primera constitución del Estado Soberano de Cartagena de 1812 al citar las cualidades para acceder a la condición de ciudadano anota en el Título 9, artículo 2:

La de ser hombre libre, vecino, padre o cabeza de familia, ó que tenga casa poblada y viva de sus rentas ó trabajo, sin dependencia de otro: y serán excluidos los esclavos, los asalariados, los vagos...., los que en su razón padecen defecto contrario al discernimiento... (Constitución de Cartagena de 1812, p. 48).

Aunque en sus primeros artículos declara la ciudadanía para todos, el peso cultural implantado en la Colonia no se hace esperar, como diría más adelante Zea (1949): «Los pueblos de América se emanciparon de España, pero no de las instituciones españolas; se alcanzó la independencia, pero no se hizo una reforma política ni un revolución social» (p. 40). Es evidente el resultado cuando los criollos se auto-reconocían como los líderes, con mayor aptitud para gobernar, dado su conocimiento de la civilización europea, según lo afirma el reformador liberal Florentino González en 1863:

Si la libertad tiene algo que esperar en estos países, es de los criollos [comprendiendo los mestizos, en que predomina la sangre europea]. Los criollos son únicamente los que han manifestado instintos favorables a la libertad y a la civilización; los que poseen las calificaciones que indican aptitud para tener parte fructuosa de la cosa pública. (Rojas, 2000, p. 61).

Siguiendo con el rastreo del uso discursivo del concepto de ciudadano encontramos que en la Constitución Nacional de 1821 y de 
acuerdo al artículo 15 de la misma, se enuncian otras características que refuerzan el interés por relacionar la función política de la educación con la construcción de ciudadanos. De esta manera la carta expone que:

Un ciudadano debía ser colombiano, ser casado o mayor de veintiún años, saber leer y escribir, pero esta condición no tendrá lugar hasta el año de 1840; Ser dueño de alguna propiedad raíz que alcance al valor libre de cien pesos. Suplirá este defecto el ejercitar algún oficio, profesión, comercio o industria útil, con casa o taller abierto sin dependencia de otro en clase de jornalero o sirviente. (Constitución de Colombia 1821).

Igualmente la Constitución de 1843 y de acuerdo al artículo número 9 declara:

\begin{abstract}
Son ciudadanos, los granadinos varones que reúnan las cualidades siguientes:

Haber cumplido la edad de veintiún años; 2. Ser dueño de bienes raíces situados en la Nueva Granada que alcancen al valor libre de trescientos pesos o tener una renta anual de ciento cincuenta pesos; y pagar las contribuciones directas establecidas por la ley, correspondientes a dichos bienes o renta; 3. Saber leer y escribir; pero esta cualidad sólo se exigirá en los que, desde primero de enero de mil ochocientos cincuenta en adelante, cumplan la edad de veintiún años. (Constitución Política de la Nueva Granada de 1843).
\end{abstract}

Más adelante la Constitución de 1863 recoge el pensamiento de los radicales declarando la ciudadanía universal y dejando que cada Estado Soberano legisle sobre el tema. Finalmente la Constitución política de 1886 declara y reconoce de la siguiente manera a los ciudadanos en sus funciones:

Artículo 15.

Son ciudadanos los colombianos varones mayores de veintiún años que ejerzan profesión, arte u oficio, o tengan ocupación lícita u otro medio legítimo y conocido de subsistencia.

Artículo 172.

Todos los ciudadanos eligen directamente Consejeros municipales y

Diputados a las Asambleas departamentales. 
Artículo 173.

Los ciudadanos que sepan leer y escribir o tengan una renta anual de quinientos pesos, o propiedad inmueble de mil quinientos, votarán para Electores y elegirán directamente Representantes (Constitución Política de Colombia de 1886).

En síntesis, la condición de ciudadano se construyó a lo largo del siglo XIX a partir de un marco de legitimación que tomaba sólo como elementos comunes la propiedad, la procedencia social, la condición civil y los oficios desempeñados de cada habitante. Las constantes en este proceso definen como se articulaban los diversos actores sociales en este imaginario de nación, sus funciones y la ubicación que tendrían por el hecho de hallarse dentro de un espacio delimitado por el territorio.

Según Conde (2011) para el año de 1811 Cartagena tenía 16.361 habitantes, más del $60 \%$ de ellos comprendidos como sectores populares bajo las categorías socio raciales de blancos pobres, mestizos, mulatos, pardos, negros y zambos; quienes compartían el entorno con el sector poblacional de dominación entendido como el conformado por españoles y criollos. Los mecanismos de relacionamiento entre las élites locales y el sector popular durante la Colonia permitieron que para el caso de la Provincia de Cartagena, estos últimos jugaran un papel fundamental en la proclamación de la Independencia absoluta de España a diferencia de otras ciudades del virreinato de la Nueva Granada. Aun así, este proceso de emancipación en el Caribe, como en la zona Andina, se inicia en condiciones de desigualdad social y política.

Las élites criollas rápidamente desplazaron de la construcción del modelo de Estado al grueso de este sector popular en cuanto a la definición, el respaldo y la defensa de derechos plenamente ganados por su participación activa y decisiva en los procesos independentistas que se realizaron. A decir lo expuesto por autores como Alfonso Múnera, las élites temían el nivel de convocatoria y acompañamiento que muchos líderes surgidos de dichos sectores provocaban en las masas, como los casos de Pedro Romero y José Prudencio Padilla (Múnera, 2005).

A este respecto Helg (1987) sostiene que Simón Bolívar y algunos otros dirigentes: 
...le temían a la movilización de los afrodescendientes libres, calificándola como pardocracia o como un potencial Haití, aun así en las pocas coyunturas en las cuales los hombres libres de color inclinaron la balanza del poder como el caso de Cartagena en noviembre de 1811 exigiendo igualdad para sí mismos pero no la abolición de la esclavitud» (Conde \& Helg, 2011, p. 29).

Para Bolívar y para todos los de su clase e incluso para los mismos pardos el ideal de libertad nunca se vínculo con el ideal de igualdad, es decir, no hubo política de emancipación sino de transmisión (Bolívar, 1984). Nuevamente la fuerza hegemónica de las élites y los dirigentes se refleja en la conciencia subalterna de los pardos los cuales no fueron conscientes de lo que significaba libertad e igualdad, lamentablemente esta disociación entre los libres de color y los esclavos se mantuvo en la gran mayoría de las nacientes repúblicas.

Con esta estructura social y política basada en el no reconocimiento mutuo se piensa la república a partir de los determinismos enmarcados por las relaciones de poder, y con ella, el tipo de educación que recibirá el nuevo ciudadano. De aquí en adelante la lucha por la emancipación se mantiene y como diría Balibar (2005, p. 20): «Toda la historia de la emancipación no es tanto la historia de la reivindicación de derechos ignorados, sino más bien la historia de la lucha real por el goce de derechos ya declarados». No se alcanzó a comprender que la libertad sin igualdad no es libertad.

De esta manera, los dirigentes y las élites nacionales y regionales decidieron los destinos de la nación arguyendo la incapacidad de la clase popular para gobernarse, negando el liderazgo político y militar de algunos pardos caribeños durante las guerras de Independencia.

Rápidamente los grupos dirigentes legaron al sistema educativo la función de garantizar el orden nacional de la mano de la Iglesia católica, para formar y dar función a su modelo de ciudadanía.

En la Constitución de Cartagena de 1812 la instrucción pública es el medio a través del cual se «iguala a los ciudadanos, les inculca y hace amables sus deberes, aumenta la propiedad individual...suaviza las costumbres y en gran manera las mejora y previene los delitos». Además, en las constituciones nacionales de 1821 y 1843 se establece que los 
ciudadanos deben saber leer y escribir para garantizar el buen ejercicio democrático. Esta exigencia como condición desaparece con el tiempo en las constituciones posteriores de 1858 y la de 1863, cobrando fuerza de otra manera, a finales del silgo XIX cuando en la constitución de 1886, se declara a Colombia como una República unitaria y a la Iglesia católica encargada de la educación de los nuevos ciudadanos para mantener «el orden social» y los ideales de hacer del territorio colombiano, una sola nación, cuyos vínculos serían: el idioma castellano, la religión católica y el conocimiento de la historia de la república; para lo que Hobsbawm (1998) califica como los elementos que caracterizan la nacionalidad política.

A esta idea de nacionalidad política, se une el lema de los conservadores: «Una nación, una raza, un Dios» (Colombia, Informe sistema nacional de Cultura. La regeneración. Organización de Estados Iberoamericanos para la Educación la ciencia y la cultura, 2008). Estos conceptos que hacen parte del imaginario político y social de la época, no incluyen a las minorías étnicas residentes a lo largo del territorio nacional y con ellos a las mujeres, los iletrados, lo vagos y todos aquellos que no hacían parte del concepto de nacionalidad. De esta manera se coloca como modelo de colombiano al ciudadano del interior, que cumple con las condiciones de ciudadanía creadas por las élites dominantes y dirigentes.

En el Departamento de Bolívar como en el resto de la región Caribe, los imaginarios de nación y de ciudadanía se trasmiten gracias a la educación, a través de manuales, cartillas textos escritos, que los niños y jóvenes que asisten a las escuelas públicas aprenden de sus maestros y maestras. En estos manuales los estudiantes aprenden a ser ciudadanos de acuerdo a las enseñanzas que vehiculizan, pues estos según Barbero, son uno de los primeros modos de ejercicio tecnológico de poder, al afirmar que: «el aprendizaje del texto (del libro-de-texto) asocia a través de la escuela un modo de transmisión de mensajes y un modo de ejercicio de poder, basados ambos en la escritura» (Barbero, 1996, p. 3). Esta asociación entre escritura y dominación, vislumbra el modelo de ciudadanía al que se aspira formar.

Por esta razón, el informe de Vicente C. Lozada de 1904, representante del Departamento de Bolívar, afirma: «En esta región se encuentran los desheredados de Colombia, el estado de la educación en el departamento es lamentable» (Gaceta Departamental de Bolívar, 1904). El político 
se refiere a "poblaciones de segunda y tercera categoría», que por su atraso industrial, hacen ver que los pueblos lejanos de las capitales y centros principales, viven desheredados del medio ambiente instruccionista. Denuncia además, que en estos pueblos existe la corrupción que robustece al gamonalismo insoportable. Sobre esto Solano, Flórez y Malkún (2010) sostiene:

Factores como la escasez y la dispersión poblacional, la pobreza y la abrumadora presencia de personas iletradas, fueron algunos de los elementos que caracterizaron a la sociedad...esta situación permitió a los letrados la concentración del poder político y que se llegara al abuso del mismo, al ser los encargados de los impuestos públicos, de las elecciones y del reparto de los impuestos. (p. 23).

En el año de 1905 en el Departamento de Bolívar existían pocas escuelas y estudiantes, para una población de 201.983 habitantes. Existían 107 planteles para un total de 7.115 estudiantes:

Tabla 1

Departamento de Bolívar

Estadística del movimiento instruccionista en el segundo semestre de 1905

\begin{tabular}{|c|c|c|}
\hline INSTRUCCIÓN PRIMARIA & VARONES & NIÑAS \\
\hline 92 oficiales & 2.988 & 3.058 \\
\hline 7 privados & 290 & 335 \\
\hline SUMA & 3.278 & 3.393 \\
\hline INSTRUCCIÓN SECUNDARIA & VARONES & NIÑAS \\
\hline 3 oficiales & 167 & 56 \\
\hline 5 privados & 326 & 36 \\
\hline Suma & 493 & 92 \\
\hline $\begin{array}{l}\text { RESUMEN } \\
107 \text { Planteles con...... } \\
\text { Población............... }\end{array}$ & $\begin{array}{l}\ldots \ldots \ldots \ldots \\
\ldots \ldots \ldots \ldots 20\end{array}$ & os \\
\hline
\end{tabular}

Fuente: Revista de Instrucción pública de Colombia (1926, p. 425).

Los datos anteriores dejan entrever el problema educativo de la región al establecer que la relación número de habitantes, número de escuelas y número de estudiantes remite directamente con problemas de índole político, económico y social. Si los letrados son los gamonales que se 
aprovechan del estado de ignorancia de los habitantes de la región que se encuentran en condiciones de pobreza y con bajo nivel escolar, cabe interrogar a lo recibido por los maestros como tradición educativa y pedagógica que reproduce los sistemas sociales, económicos y políticos.

Lo recibido al pasar por la conciencia del maestro toma su forma y adquiere otro matiz, que puede ser mejorado de acuerdo al modo de estar en esta realidad que recibe y en la cual va a dejar sus sueños, su tiempo, sus energías, todo su ser en la tarea de construir con otros la realidad del presente. Es cuestionarse acerca del concepto de ciudadanía para posibilitar un nuevo concepto que traspase las ideas de territorialidad y sitúe a la nueva ciudadanía en contextos de emancipación y reclame esos derechos que todos conocen pero que nadie quiere reconocer: el derecho ciudadano que otorga el simple hecho de ser persona humana, sin más en cualquier lugar del mundo.

Si durante todo el tiempo las discusiones sobre ciudadanía han girado alrededor de dos temas ¿Quién tiene derecho a la ciudadanía? y ¿Qué acarrea para un ciudadano ejercer la ciudadanía? Es en este tiempo y hora donde el ser humano puede recuperar su condición ciudadana sin más restricciones.

\section{Momento continuante}

Siguiendo a Ellacuría (1991), y como diría Palacios (2005), nos ha sido entregada una educación fragmentada, de la cual los maestros deben dar cuenta. En el Departamento de Bolívar la hegemonía de la tecnología que arroja el dominio de la lectura y la escritura sigue estando en manos de unos pocos. Los procesos educativos han mantenido por largos años las diferencias raciales, políticas y sociales. Entonces: ¿Qué hemos hecho con lo recibido de los procesos educativos? Tristemente excluir, ser indiferentes ante diversas realidades, ignorar, desconocer e incomprender al otro.

Tal y como afirma Gadamer (2007, p. 50):

Lo que interesa al conocimiento histórico no es el saber cómo los hombres, los pueblos, los Estados se desarrollan en general, sino, por el contrario, cómo este hombre, este pueblo, este Estado, ha llegado a ser lo que es; cómo esto ha podido pasar y llegar a suceder allí. 
En este sentido para que los contenidos de la educación no se estanquen en el tiempo y se sigan repitiendo los esquemas del miedo, la ignorancia y la indiferencia que se han convertido en las características de las nuevas generaciones, los maestros deben interrogarse para recrear lo dado en las preguntas de discusión y diálogo del: ¿Qué hacer? ¿Cómo hacer? Y ¿Para qué educar?

\section{Momento progrediente. Lo entregado}

Por tanto, investigar la formación del ciudadano en las escuelas públicas del Departamento de Bolívar, durante la hegemonía conservadora a comienzos del siglo XX, es una oportunidad para identificar la prolongación de un modelo de sistema político, económico y social que ha determinado la manera como se educan y se construyen los sujetos en el devenir de la historia. Pero, muchos dirían ¿Para qué?, si eso ya pasó. Tal vez una posible respuesta sería: para conocer e interpretar la realidad política, social, educativa y pedagógica de la época, que temo afirmar, aún permanece en cada uno de nosotros.

La realidad educativa y pedagógica al pasar por la conciencia histórica del maestro es interpretada, es decir, que lo entregado es la interpretación de la realidad que se constituye en el elemento progrediente del cual cada uno es responsable.

La realidad según Zubiri [2007) es posibilidad; es historia que se renueva en cada conciencia del ser que trabaja, en cada ser que actúa sobre la realidad interpelada por los contextos sociales, políticos, económicos, pedagógicos, y educativos que afronta en la cotidianidad de su existencia. Es necesario entonces entender la pedagogía como saber pedagógico (Zuluaga, 1999) que supera el discurso de la hegemonía dominante para construir la realidad desde las situaciones de negación, exclusión, indiferencia, ignorancia y no reconocimiento de la diversidad y la diferencia propia, de una nación que se construyó en el ideal imaginado de la totalidad.

Las ciencias del Espíritu en su nueva interpretación epistemológica permiten comprender que la Historia de la Educación es el resultado de las prácticas pedagógicas del maestro. Esto nos indica que es el momento de dar razón de nuestras prácticas de aula a través de las cuales se está construyendo la nueva realidad educativa y pedagógica de la región Caribe colombiana.

ITINERARIO EDUCATIVO • ISSN OISI-2753 • AÑO XXVI, N. ${ }^{\circ} 60$ • ENERO - JUNIO DE ZOIS • P. 97-II6 
Por otro lado, la realidad educativa sigue arrojando un modelo de ciudadano y ciudadanía que reproduce al hombre común, que sale de nuestras escuelas sin mayores pretensiones de transformación.

La invitación entonces es pensar la pedagogía y la educación como un proceso de humanización, reconocer que cada maestro y maestra hace historia y es responsable de ella. Por consiguiente, se podría optar por trabajar día a día en el rescate y el reconocimiento del ser humano, como único requisito para dar razón de su condición de ciudadano, no sólo de un territorio nacional definido sino de cualquier espacio en el que se encuentre como ciudadano del mundo.

Una nueva teoría de la ciudadanía debe ser desarrollada con el fin de dar cuenta de los crecientes movimientos transnacionales subordinados y de los desafíos que presenta el régimen internacional de los derechos humanos. La ciudadanía debe ser desterritorializada (menos nacional y más igualitaria), de tal manera que la diáspora jurídica de millones de personas desplazadas pueda llegar a su fin. La ciudadanía debe ser «descanonizada» (menos sagrada y más democrática), de manera tal que el pasaporte y la visa dejen de ser un fetiche jurídico de acuerdo con el cual la vida cambia y del cual depende la dignidad humana de muchas personas» (Santos, 1998, p. 147).

\section{Referencias}

Balibar, É. (2005). Violencias, Identidades y Civilidad. Para una cultura política global. 15-21. Barcelona: Gedisa. Editorial.

Barbero, J. M. (1996). Heredando el futuro. pensar la educación desde la comunicación.» En Nómadas, Colombia número 5, 1996: 1 -14.

Bloch, M. (1997). Introducción a la Historia. Bogotá, Colombia: Fondo de Cultura Económica.

Bolívar, S. (1984). Simón Bolivar escritos politicos . Bogotá: El Ángora.

Constitución de Cartagena de 1812. Recuperado de http://www.banrepcultural.org.» http://www.banrepcultural.org/sites/default/files/89670/ brblaa169729.pdf. 01 de 01 de 1812. http://www.banrepcultural.org/ sites/default/files/89670/brblaa169729.pdf.

Constitución política de la Nueva Granada de 1843. Recuperado de HYPERLINK «https://ch1prd0202.outlook.com/owa/redir.aspx?C=GujJI 
1NWgEe2KeTBiUfAyFs50LbMi88IxVZu0yy4_qa_x_k07m7_bNVvN FTvv9Ks99SOSKIgTsk.\&URL=http\%3a\%2f\%2fwww.alcaldiabogota. gov.co\%2fsisjur\%2fnormas\%2fNorma1.jsp\%3fi\%3d13695» \t «_blank» www.alcaldiabogota.gov.co/sisjur/normas/Norma1.jsp?i=13695

Colombia, Informe sistema nacional de Cultura. (2008). La regeneración. Organización de Estados Iberoamericanos para la Educación la ciencia y la cultura.

Conde, J. y Helg, A. (2011). Padilla Libertador del Caribe Colombiano. Cartagena: Ediciones Unitecnológica.

Ellacuría, I. (1991). Filosofía de la realidad histórica. Madrid: Trotta, S.A.

----. (1985). Función Liberadora de la filosofía, escritos políticos I. Madrid: Trotta, S.A.

Freire, P. (2005). Pedagogía del Oprimido. En Pedagogia del Oprimido. México: Siglo XXI Editores.

Gaceta Departamental de Bolívar, Cartagena, diciembre 22 de 1903

Gaceta Departamental de Bolívar, Cartagena, noviembre 5 de 1904

Gadamer, H. G. (2007). El problema de la conciencia histórica. Madrid: Tecno.

----_- (2010). Verdad y Método II. Salamanca, España: Ediciones Sígueme.

Helg, A. (1987). La educación en Colombia: 1918- 1957. Bogotá: Plaza \& Janés.

Hobsbawm, E. (1998). Naciones y nacionalismo desde 1780. Barcelona: Critica Grijalbo Mondadori S.A.

Revista de Instrucción pública de Colombia, Bogotá, Marzo 1926 Tomo VXIII

König, H-J. (1994). En el camino hacia la nación. Bogotá: Banco de la República.

Múnera, A. (2005). Fronteras Imaginadas. La construcción de las razas y de la geografía en el siglo XIX colombiano. Colombia: Planeta.

Palacios, M. (2005). Entre la legitimidad y la violencia Colombia 1875 1994. Bogotá: Grupo Editorial Norma.

Revista de Instrucción pública de Colombia, Bogotá, Marzo 1926 Tomo VXIII 
Rojas, M. C. (2000). La economía política de la civiliazación. En Revista de estudios sociales. Universidad de los Andes, pp. 61 -70.

Santos, B. (1998). La globalización del derecho. Los nuevos caminos de la regulación y la emancipación. Bogotá, Colombia: ILSA, Universidad Nacional.

Silva, R. (2005). La Ilustración en el virreinato de la Nueva Granada, estudios de historia social. Medellín: La carreta editores.

Solano, S. P., Flórez, R. \& Malkún, W. (2010). Ganadreos y comerciantes: el manejo del poder político en el estado soberano de Bolívar (Colombia) 1857 -1886. En Historia y sociedad n¹. Medellín: Universidad Nacional. pp.15-42.

Zea, L. (1949). Dos etapas de pensamiento en Hispanoamérica. Del romanticismo al positivismo . México: El Colegio de México.

Zubiri, X. (2007). Antropología y ontología. En Escritos menores (19531983). Madrid, España: Alianza Editorial Fundación Xavier Zubiri.

Zuluaga, O.L. (1999). Pedagogía e historia, la historicidad de la pedagogía. La enseñanza, un objeto de saber. Bogotá, Colombia: Siglo del Hombre Editores, Anthropos, Editorial Universidad de Antioquia. 